\title{
Data Driven Design Optimization Methodology A Dynamic Data Driven Application System
}

\author{
Doyle Knight \\ Dept of Mechanical and Aerospace Engineering \\ Rutgers - The State University of New Jersey \\ New Brunswick, NJ 08903 \\ knight@soemail.rutgers.edu
}

\begin{abstract}
Engineering design optimization using concurrent integrated experiment and simulation is a Dynamic Data Driven Application System (DDDAS) wherein remote experiment and simulation can be synergistically utilized in real-time to achieve better designs in less time than conventional methods. The paper describes the Data Driven Design Optimization Methodology (DDDOM) being developed for engineering design optimization.
\end{abstract}

\section{Introduction}

A Dynamic Data Driven Application System (DDDAS) is an application software system capable of accepting and effectively utilizing remote data in real time (i.e., during the execution of the application software). Many software systems currently utilize static input data, i.e., input data which is specified $a$ priori. The key concept of DDDAS is the generalization of application software systems to dynamically utilize real-time data arising from remote experiment and simulation, and to control such remote experiment and simulation to improve the performance of the application software system. The concept is illustrated schematically in Fig. 1.

Dynamic Data Driven Application Systems have become a major subject of research interest due to the continuing rapid advances in technology. These include, for example, improvements in computer processor performance (i.e., doubling every 18 to 24 months (Berkowitz 1996)), network bandwidth, Rapid Prototyping (RP) and real-time data acquisition and control (e.g., using MicroElectro Mechanical Systems (MEMS)). These rapid technological advances provide the means to revolutionize the use of existing and future application software systems. For example, the NSF TeraGrid ${ }^{1}$ is a multi-year effort to deploy 20 TFlops of computing capacity at five sites connected through a 40 Gbps network. The TeraGrid will offer unprecedented remot computing capability which can be utilized in real-time by application software systems executing at users' sites. The incorporation of the NSF Teragrid into an application software system is therefore an example of a Dynamic Data Driven Application System.

${ }^{1}$ http://www.ncsa.uiuc.edu/About/TeraGrid/ 


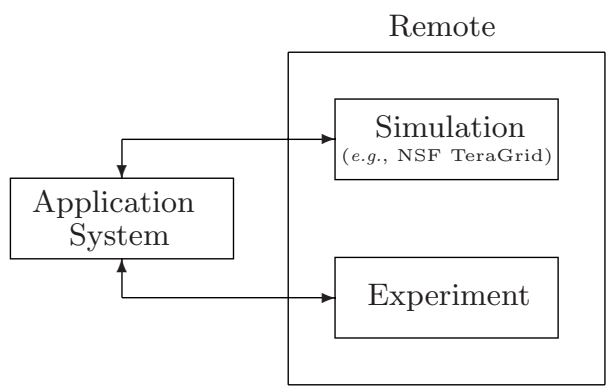

$\longleftarrow$ Remote real-time data to Application System

$\longrightarrow$ Control by Application System

Fig. 1. Use of remote experiment and simulation in application software

The objective of this paper is to present a Dynamic Data Driven Application System for engineering design optimization. In the following sections, we describe the general engineering design optimization problem, the conventional approach to engineering design optimization, our Dynamic Data Driven Application System for engineering design optimization, and a demonstration example.

\section{Design Optimization}

The general engineering design optimization problem is defined as

$$
\operatorname{minimize} f_{j}(\boldsymbol{x}) \text { for } j=1, m
$$

subject to

$$
d_{k} \leq 0 \quad \text { for } k=1, l
$$

where

$$
\boldsymbol{f}=\left(f_{1}, \ldots, f_{m}\right)^{\mathrm{T}}
$$

is the vector of objective functions and

$$
\boldsymbol{d}=\left(d_{1}, \ldots, d_{l}\right)^{\mathrm{T}}
$$

is the vector of constraints and

$$
\boldsymbol{x}=\left(x_{1}, \ldots, x_{n}\right)^{\mathrm{T}}
$$

is the vector of design variables. The solution to the general engineering design problem is the set of non-dominated designs known as the Pareto Set (Deb 2001). Within this set of designs, improvement in one design criterion $f_{j}$ can only be achieved at the expense of the degradation in the performance of one or more other design criteria. 


\section{Conventional Engineering Design Optimization}

Engineering design is a potential Dynamic Data Driven Application System wherein remote real-time input from high performance computing resources (e.g., NSF Teragrid) and experiment (e.g., using capability for rapid fabrication of new experimental configurations) can produce a better design in shorter time with lower cost than the conventional approach. Fig. 2 illustrates the conventional approach to engineering design. An initial design is typically evaluated using simulation software executing on local computer resources. An example is Computational Fluid Dynamics (CFD) simulation using one of the several available commercial CFD software packages (e.g., FLUENT, GASP, STAR-CD). The results of the simulation are reviewed by the designer and additional simulations performed as needed. An experiment is then planned and executed, and the results compared with the simulation. The process is iterated until a satisfactory design has been achieved. A major limitation of the conventional approach is the absence of synergistic real-time use of remot experimental data and high performance computer resources (e.g., the NSF TeraGrid).

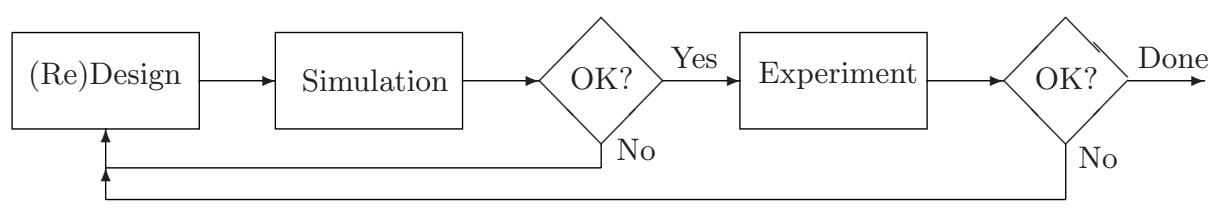

Fig. 2. Conventional approach to engineering design

\section{Data Driven Design Optimization Methodology}

We are developing an integrated software system, denoted Data Driven Design Optimization Methodology (DDDOM), to synergistically incorporate remote experiment and simulation into an automated design optimization methodology. The DDDOM is comprised of six elements as illustrated in Fig. 3 and are summarized below.

Controller The DDDOM Controller provides direction and control of the design optimization process. It is written in Perl (Wall et al 2000), a powerful systems programming language which enables robust program control on local and remote systems. The Controller utilizes the Globus software system (Foster and Kesselman 1997) to link to the NSF TeraGrid.

User Interface The User Interface provides for the initiation and monitoring of the design optimization. It also provides visualization of the intermediate results of the design optimization process. The interface is written in Perl/Tk (Lidie and Walsh 2002). Both Perl and Perl/Tk operate on virtually every platform and operating system. 


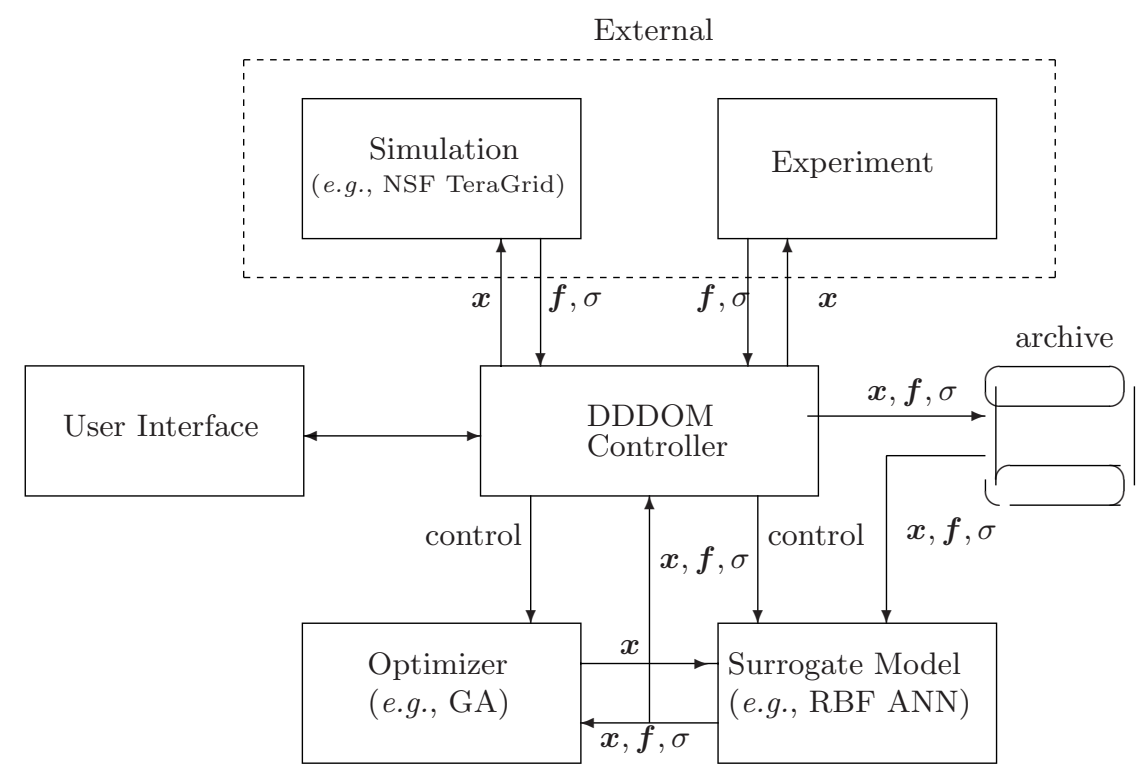

Fig. 3. Data Driven Design Optimization Methodology

Optimizer A Multi-Criteria Design Optimization (MDO) algorithm is used to search the design space to define the Pareto Set. Both local (i.e., gradient-based) and global (i.e., stochastic-based) algorithms are used. Examples are CFSQP (Lawrence et al 1994) and GADO (Rasheed and Hirsh 1999), respectively.

Surrogate Model Surrogate Models of the objective functions $\boldsymbol{f}$ are developed during the design optimization using experiment and simulation. These models are utilized by the optimizer to search the design space for the Pareto Set. We utilize Response Surfaces (Myers and Montgomery 1995) and Radial Basis Function Artificial Neural Networks (Hertz et al 1991). The Surrogate Model incorporates the measure of uncertainty $\sigma$ in the results generated by the experiment and simulation.

Experiment Experiments are performed in real-time with the conditions of the experiment defined by the Controller. The interface with experiments is typically National Instruments LabView (Travis 2002). Rapid Prototyping is used to build new models as needed with minimal delay (e.g., one day).

Simulation Simulations can be performed locally (i.e., at the same site as the DDDOM is operated) or remotely (e.g., on the NSF TeraGrid). The simulations are performed in real-time with the conditions of the simulation defined by the Controller.

\section{Example}

A five minute demonstration has been developed to illustrate the principle of operation of the DDDOM. The limited duration of the demonstration precludes 
the actual use of RP which typically requires several hours for fabrication of the prototype for experiment. Therefore, we have selected a single experimental configuration. Nonetheless, the experiment provides data to the DDDOM Controller in real time, and the Controller directs the experiment, thereby illustrating the DDDOM concept.

The specific problem is defined as follows. Consider the flow of air in a converging-diverging nozzle attached to a stagnation chamber as illustrated in Fig. 4. The nozzle is designed to achieve Mach 2 at the exit assuming isentropic expansion throughout the nozzle. Depending upon the ratio of stagnation pressure $p_{t_{\infty}}$ to ambient pressure $p_{a}$, the flow in the diverging section of the nozzle can be 1) subsonic everywhere, 2) supersonic and subsonic with a normal shock at some location in the diverging section, and 3) supersonic everywhere (Anderson 2003). In the latter case, the theoretical exit Mach number is two. A one-dimensional simulation based upon inviscid gas dynamics (Liepmann and Roshko 1957) indicates that the exit pressure ratio $p_{e}$ vs stagnation pressure $p t_{\infty}$ behaves as shown by the curve denoted Simulation in Fig. 5 . This behavior is idealized, however, due to the neglect of the viscous boundary layer on the nozzle walls and the effect of the interaction of the normal shock wave (if it exists) with the boundary layers. Consequently, the actual exit pressure $p_{e} v s$ stagnation pressure $p_{t_{\infty}}$ curve differs substantially from the idealized shape.

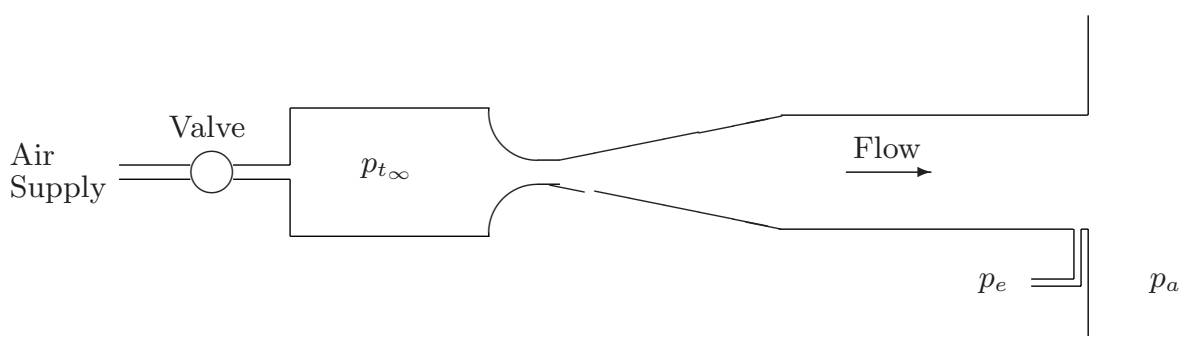

Fig. 4. Experimental configuration

The objective of the optimization is to determine the stagnation pressure $p_{t_{\infty}}$ which yields the minimum exit pressure $p_{e}$ for a fixed ambient pressure $p_{a}$. Although this is a single design objective problem, it illustrates the fundamental concepts of DDDOM. Due to the physical assumptions inherent in the simulation code, the $p_{e}$ vs $p_{t_{\infty}}$ function obtained from the simulation can be significantly in error. This is an intentional choice to force the DDDOM Controller to build and refine the Surrogate Model utilizing both simulation and experimental data to determine the optimum value of $p_{t_{\infty}}$ (i.e., the value of $p_{t_{\infty}}$ which yields the minimum value of $p_{e}$ ). The dynamic updating of the Surrogate Model using real time experimental data is an example of a Dynamic Data Driven Application System. 


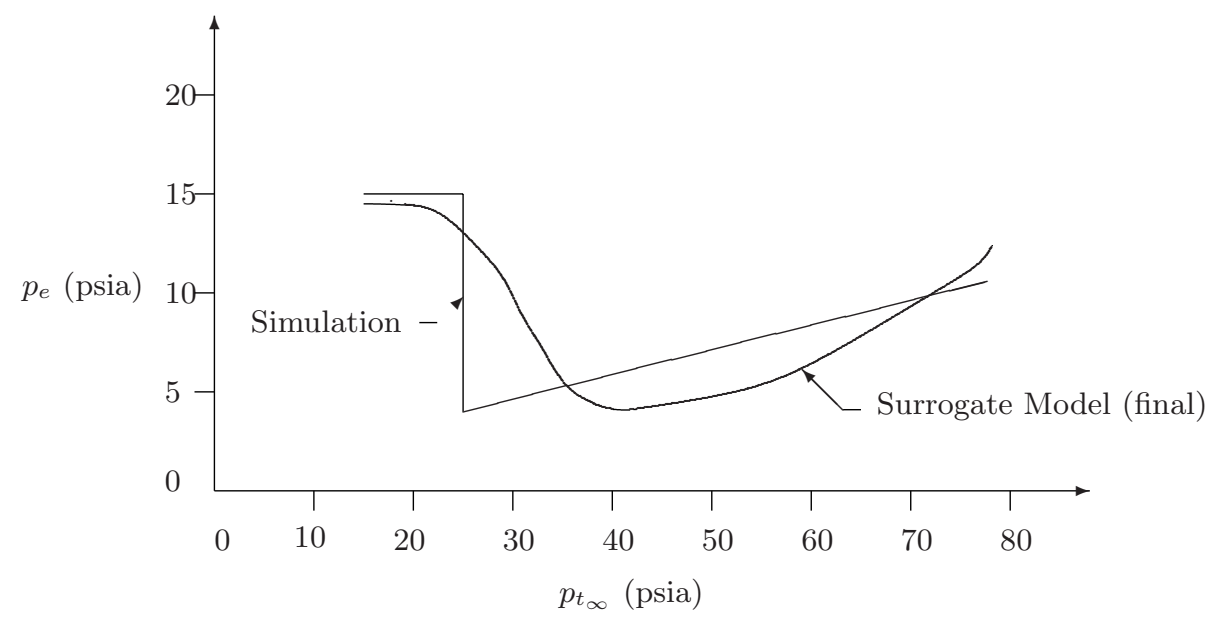

Fig. 5. Exit pressure $p_{e} v s$ stagnation pressure $p_{t_{\infty}}$

The experiment is located in a laboratory in the Department of Mechanical and Aerospace Engineering at Rutgers University and controlled by a workstation running Windows 98 . The DDDOM is executed on a separate workstation in another building running Linux. The DDDOM and experiment workstation communicate via high speed Ethernet. A National Instruments LabView Virtual Instrument (VI) program (Travis 2002) executes on a workstation adjacent to the experiment. The VI continually monitors an input file on the workstation which contains a specified value of stagnation pressure $p_{t_{\infty}}$ and adjusts the stagnation pressure by means of a servo-controlled valve to the specified value. The VI thereafter writes the measured values of the stagnation pressure $p_{t_{\infty}}^{\prime}$ (which differs from the specified $p_{t_{\infty}}$ due to "play" in the valve) and exit pressure $p_{e}^{\prime}$ on a file on the workstation.

The DDDOM Controller (Fig. 3) manages the optimization problem by the following sequence:

1. The Surrogate Model is initialized using the one-dimensional inviscid simulation code.

2. The optimizer (a Genetic Algorithm) determines the minimum of the $p_{t_{\infty}} v s$ $p_{e}$ curve using the current Surrogate Model.

3. A value of $p_{t_{\infty}}$ is chosen for the experiment. In the early stages of the optimization, the value determined in step no. 2 is chosen with a probability of 0.5 ; otherwise, a random value is chosen within the overall range of experimentally possible values of $p_{t_{\infty}}$ (i.e., 15 psi to $60 \mathrm{psi}$ ). This procedure avoids premature convergence to an exit pressure which is not the actual minimum.

4. A file is transferred to the workstation containing an identification number and the prescribed value of $p_{t_{\infty}}$.

5. The VI on the workstation reads the file, changes the experimental total pressure to the specified value within experimental tolerances, and writes 
the identification number, the actual experimental total pressure $p_{t_{\infty}}^{\prime}$ and exit pressure $p_{e}^{\prime}$ to the file.

6. The DDDOM Controller accesses the file and reads the experimental values of $p_{t_{\infty}}^{\prime}$ and $p_{e}^{\prime}$. The database for the Surrogate Model is updated using this data and the nearest value to $p_{t_{\infty}}$ in the database which was generated by the one-dimensional simulation code is removed.

7. The convergence of the Surrogate Model is checked. If it is not converged, the procedure is repeated beginning at step no. 2 .

\section{Conclusions}

Engineering design is an example of a Dynamic Data Driven Application System wherein real-time data from experiment and simulation can be effectively utilized to yield better designs in shorter time. A Data Driven Design Optimization Methodology (DDDOM) is being developed which incorporates experiment and simulation in a real-time, synergistic manner. The theory and organization of DDDOM is described. An example demonstration is described.

\section{Acknowledgments.}

The research is sponsored by the US National Science Foundation under grant CTS-0121058. The program managers are Drs. Frederica Darema, C. F. Chen and Michael Plesniak. The assistance of Profs. Greg Elliott and Madara Ogot in development and implementation of the supersonic nozzle experiment is gratefully acknowledged.

\section{References}

Anderson, J. Modern Compressible Flow with Historical Perspective. McGraw Hill (2003), New York.

Berkowitz, B. Information Age Intelligence. Foreign Policy 103 (1996) 35-50.

Deb, K. Multi-Objective Optimization using Evolutionary Algorithms. John Wiley \& Sons (2001), New York.

Foster, I. and Kesselman, C. Globus: A Metacomputing Infrastructure Toolkit. International Journal of Supercomputer Applications 11 (2) (1997) 115-128.

Hertz, J., Krogh, A., and Palmer, R. Introduction to the Theory of Neural Computation. Lecture Notes Vol I, Santa Fe Institute Studies in the Sciences of Complexity. Perseus Publishing (1991), Cambridge, MA.

Knight, D., Elliott, G., Jaluria, Y., Langrana, N., and Rasheed, K. Automated Optimal Design Using Concurrent Integrated Experiment and Simulation. AIAA Paper No. 2002-5636, AIAA/ISSMO Symposium on Multidisciplinary Analysis and Optimization, Atlanta, GA (2002).

Lawrence, C., Zhou, J., and Tits, J. User's Guide for CFSQP Version 2.1. University of Maryland, Electrical Engineering Dept \& Institute for Systems Research (1994).

Lidie, S., and Walsh, N. Mastering Perl/Tk. O'Reilly and Associates (2002), Sebastopol, CA. 
Liepmann, H., and Roshko, A. Elements of Gas Dynamics. John Wiley \& Sons (1957), New York.

Myers, R., and Montgomery, D. Response Surface Methodology - Process and Product Optimization Using Design Experiments. John Wiley \& Sons (1995), New York.

Rasheed, K., and Hirsh, H. Learning to be Selective in Genetic-Algorithm-Based Design Optimization. Artificial Intelligence in Engineering, Design, Analysis and Manufacturing 13 (1999) 157-169.

Travis, J. LabVIEW for Everyone. Prentice Hall (2002), Upper Saddle River, NJ.

Wall, L., Christiansen, T., and Orwant, J. Programming Perl. O'Reilly and Associates (2000), Sebastopol, CA. 